\title{
Diacronie
}

Studi di Storia Contemporanea

$N^{\circ} 33,1 \mid 2018$

Guerra e pace

\section{Uscire dalla catastrofe}

La città di Napoli fra guerra aerea e occupazione alleata

\section{Martina Gargiulo}

\section{(2) OpenEdition \\ Journals}

Edizione digitale

URL: http://journals.openedition.org/diacronie/7187

DOI: $10.4000 /$ diacronie. 7187

ISSN: 2038-0925

Editore

Association culturelle Diacronie

Notizia bibliografica digitale

Martina Gargiulo, « Uscire dalla catastrofe », Diacronie [Online], N3 33, 1 | 2018, documento 2, Messo

online il 29 mars 2018, consultato il 06 mai 2019. URL : http://journals.openedition.org/diacronie/7187 ; DOI : 10.4000/diacronie.7187

Creative Commons License 


\title{
Diacronie
}

Studi di Storia Contemporanea

\section{$33,1 / 2018$}

Guerra e pace: declinazioni politiche, sociali e culturali del conflitto in età contemporanea

\section{Uscire dalla catastrofe. La città di Napoli fra guerra aerea e occupazione alleata}

\author{
Martina GARGIULO
}

Per citare questo articolo:

GARGIULO, Martina, «Uscire dalla catastrofe. La città di Napoli fra guerra aerea e occupazione alleata», Diacronie. Studi di Storia Contemporanea : Guerra e pace: declinazioni politiche, sociali e culturali del conflitto in età contemporanea, 33 , $1 / 2018,29 / 03 / 2018$

URL: < http://www.studistorici.com/2018/03/29/gargiulo_numero_33/ >

Diacronie Studi di Storia Contemporanea $\rightarrow$ http://www.diacronie.it

Rivista storica online. Uscita trimestrale.

redazione.diacronie@hotmail.it

Comitato di direzione: Naor Ben-Yehoyada - João Fábio Bertonha - Christopher Denis-Delacour - Maximiliano Fuentes Codera Anders Granås Kjøstvedt - John Paul Newman - Deborah Paci - Niccolò Pianciola - Spyridon Ploumidis - Wilko Graf Von Hardenberg

Comitato di redazione: Jacopo Bassi - Luca Bufarale - Gianluca Canè - Luca G. Manenti - Fausto Pietrancosta - Alessandro Salvador - Matteo Tomasoni - Luca Zuccolo 


\title{
2/ Uscire dalla catastrofe. La città di Napoli fra guerra aerea e occupazione alleata
}

\author{
Martina GARGIULO
}

Questo articolo costituisce una prima rilettura, in chiave locale, della congiuntura storica del secondo conflitto mondiale e in particolar modo degli effetti sul piano materiale e morale scaturiti dai bombardamenti su larga scala dei centri abitati. Il processo di sdoganamento dei raid aerei contro la popolazione nei termini di un lecito strumento di guerra affondava le proprie radici dall'esigenza di rompere la "catena di solidarietà" che legava il fronte interno ai soldati impegnati nei combattimenti. Lo scoppio del nuovo conflitto mondiale coincise con una forte saldatura fra la pianificazione degli attacchi aerei su larga scala e il perfezionamento dei dettami della guerra psicologica. Durante la prima fase del conflitto, le città meridionali furono duramente colpite dalle campagne di bombardamento inglesi, a cui sarebbe seguite di lì a poco quelle americane. Per la sua posizione strategica e per il ruolo giocato dal porto, Napoli subì una lunga serie di rovinosi attacchi, con gravi danni inflitti all 'intero tessuto urbano e in particolare al patrimonio artistico. Con l'ingresso degli eserciti alleati in città e la fine anticipata delle ostilità belliche si aprì per la popolazione napoletana una nuova fase della sua storia, che non coincise tuttavia con un miglioramento immediato delle sue condizioni di vita. Il problema abitativo rappresentava allo stesso tempo la prima emergenza da affrontare e una sfida contro il tempo aggravata dalle parallele requisizioni a tappeto messe in moto dalle nuove forze occupanti.

Elenco delle abbreviazioni degli archivi citati

ACS $=$ Archivio Centrale dello Stato

AIL $=$ Archivio Istituto Luce

$\mathrm{ASNa}=$ Archivio di Stato di Napoli

\section{Le campagne di bombardamenti}

Il secondo conflitto mondiale apportò un cambiamento radicale nel modo di concepire la guerra, la quale assunse pienamente una dimensione totalizzante. I bombardamenti a tappeto sulle città, per il loro visibile impatto in termini di vite umane e pressione psicologica adoperata, 
ne costituirono l'esempio meglio noto e concreto ${ }^{1}$. L'intera nazione nemica costituiva un obiettivo di guerra, compresi i confini interni delle aree abitate, centri urbani o campagne che fossero. La linea del fronte finì così con l'abbracciare l'intera conformazione geografica dello Stato nemico. $\mathrm{A}$ segnare il cambio di rotta era stata in primo luogo la rinnovata esigenza di indebolire e disarticolare la rete del fronte interno, e di minarne ogni azione di supporto morale e materiale ai soldati impegnati nei combattimenti. Attraverso la duplice strategia di pianificazione e legittimazione dei bombardamenti aerei nei termini di un lecito strumento di guerra, il secondo conflitto mondiale aprì due fasi di rottura: sia con le recidive ritrosie morali circa l'opportunità di infierire sulla popolazione inerme; sia con il processo di esternalizzazione dai confini europei delle dinamiche belliche e dell'uso indiscriminato dei suoi strumenti distruttivi.

L'intenzione di bombardare l'Italia era stata accarezzata dalle forze alleate prima del suo ingresso ufficiale in guerra, il 10 giugno 1940. In previsione della riorganizzazione dei comparti bellici italiani a fianco della Germania, nel mese di maggio inglesi e francesi avevano pianificato una massiccia offensiva aerea, nelle ore diurne e notturne, contro le città più importanti. I punti deboli italiani erano ben noti e dovevano essere colpiti: bisognava trarre il massimo vantaggio sia dalla contraerea scarsamente equipaggiata, sia dall'impreparazione tattico-militare, sia dalla vulnerabilità del tessuto economico e sociale ${ }^{2}$. La caduta francese non arrestò i propositi inglesi di mettere in ginocchio gli stabilimenti industriali e le infrastrutture nemiche. L'alto rischio per i bombardieri di sorvolare i cieli francesi, strettamente controllati dall'occupante tedesco, condizionò in modo preponderante la decisione di colpire, durante la prima fase del conflitto, i centri urbani dell'Italia meridionale, sfruttando le basi in dotazione a Malta ${ }^{3}$.

I raid sul Meridione colpirono incessantemente già dal 1940. Fra le città più colpite figurò Napoli, seguita da Palermo, Bari, Taranto, Catania, Messina, Trapani, Augusta, Cagliari, Brindisi, Crotone. Il numero di vittime e di danni arrecati in una fase del conflitto al suo stadio iniziale fu notevole, tanto che all'interno del recente dibattito storiografico si comincia a parlare di

\footnotetext{
${ }^{1}$ La complessità del fenomeno della guerra aerea, specie nei termini di percezione del conflitto stesso e di formazione della memoria collettiva da parte delle popolazioni coinvolte, ha costituito uno stimolo proficuo per numerosi studi che, con diversi tagli e sensibilità, si sono gradualmente approcciati alla questione. Per la Gran Bretagna, si segnalano in particolare: BIDDLE, Tamis Davis, Rhetoric and Reality in Air Warfare: The Evolution of American and British Ideas about Strategic Bombing 1914-1945, Princeton, Princeton University Press, 2002; JONES, Helen, British civilians in the front line: air raids, productivity and wartime culture 1939-1945, Manchester, Manchester University Press, 2006. Ricche disamine in chiave comparativa, specie in rapporto al caso tedesco e francese, sono invece contenute in: BALDOLI, Claudia, KNAPP, Andrew, OVERY, Richard (eds.), Bombing, States and Peoples in Western Europe 1940-1945, London, Continuum, 2011; BALDOLI, Claudia, KNAPP, Andrew, Forgotten Blitzes: France and Italy under Allied Air Attack, 1940-1945, London, Bloomsbury, 2012; OVERY, Richard, The Bombing War. Europe 1939-1945, London, Penguin, 2013.

${ }^{2}$ GRIBAUDI, Gabriella, «L'impatto della guerra aerea sulla popolazione civile», in Storia e memoria, 1, 2013, pp. 39-68, p. 39.

${ }^{3}$ Ibidem, p. 40.
} 
"questione meridionale dei bombardamenti» ${ }^{4}$. Nel caso di Napoli, l'intenzione di colpire in questa fase del conflitto "solo" le aree considerate strategiche non risparmiò la stazione e gli stabilimenti industriali della zona est della città, specialmente le raffinerie, dove divamparono di conseguenza spaventosi incendi ${ }^{5}$. Nel gennaio del 1941 le incursioni si spinsero fin dentro il centro urbano, in quartieri densamente popolati. Furono colpite la sede del Banco di Napoli in via Toledo e gli edifici del Rione Carità; nel mese di luglio venne presa di mira una vasta area a est e a ovest di via Roma, che provocò distruzioni alle abitazioni dei Quartieri Spagnoli e a via Medina.

Le bombe del 1940-41 mieterono le prime ferite mortali al patrimonio artistico della città, che negli anni successivi sarebbe stato ulteriormente danneggiato. A soffrirne fu la facciata della Chiesa dei Santi Francesco e Matteo sita in vico Lungo San Matteo (Quartieri Spagnoli) e il Teatro dei Fiorentini in via Fiorentini (Rione Carità), che perse l'intera copertura anti-aerea ${ }^{6}$. Di pari passo allo sgancio di nuovi ordigni gli inglesi sperimentarono la strategia della guerra psicologica, lanciando sui cieli di Napoli i primi volantini che invitavano la popolazione a boicottare il rifornimento delle navi tedesche dirette in Nord Africa.

Napoletani! Noi inglesi, che mai fummo in guerra con voi, vi mandiamo questo messaggio [...] Noi vogliamo solo la pace con voi. Ma siamo costretti a bombardare la vostra città perché voi permettete ai tedeschi di servirsi del vostro porto. Finché partono da Napoli navi cariche di armi e materiali tedeschi per le forze germaniche in Libia, Napoli sarà ripetutamente bombardata?

Durante l'inverno del 1941 la popolazione napoletana ebbe la chiara percezione che lo scenario di una guerra-lampo, loro promessa, non si sarebbe realizzata e che le difese anti-aeree presenti sul territorio fossero drammaticamente insufficienti. Emergeva dunque in tutta la sua drammaticità il problema dei ricoveri: insufficienti per una città densamente urbanizzata come Napoli, e di conseguenza sovraffollati ed estremamente carenti di aria e di luce. La stessa conformazione geografica della città, stretta fra le colline e la fascia costiera, e il pochissimo spazio disponibile nelle strade-alveare dei suoi quartieri costituirono la maggiore ipoteca per la costruzione di rifugi che potessero definirsi sufficientemente sicuri. Vennero sfruttate le risorse

\footnotetext{
${ }^{4}$ GIOANNINI, Marco, Bombardare l'Italia. Le strategie alleate e le vittime civili, in LABANCA, Nicola (a cura di), I bombardamenti aerei sull'Italia, Bologna, Il Mulino, 2012, pp. 79-100, pp. 84-86.

${ }^{5}$ GRIBAUDI, Gabriella, «L'impatto della guerra aerea», cit., p. 40.

${ }^{6}$ PANE, Andrea, Danni bellici, restauri e ricostruzioni a Napoli tra Quartieri Spagnoli, Monteoliveto e Rione Carità, in CASIELLO, Stella (a cura di), Offese di guerra. Ricostruzione e restauro nel Mezzogiorno d'Italia, Firenze, Alinea editrice, 2011, pp. 73-101, pp. 75-76.

${ }^{7}$ Archivio di Stato di Napoli (d'ora innanzi ASNa), Prefettura, Gabinetto, secondo versamento, b. 1222/1, Disciplina di guerra. Bombardamenti (1941-1942), foglio volante della RAF, luglio 1941. Vedi anche GRIBAUDI, Gabriella, Guerra totale. Tra bombe alleate e violenze naziste. Napoli e il fronte meridionale 1940-1944, Torino, Bollati Boringhieri, 2005, pp. 53-54; PANE, Andrea, op. cit., p. 78.
} 
già presenti, come i tunnel delle ferrovie e della metropolitana e le cavità del sottosuolo, meglio note con l'appellativo di "Napoli sotterranea": un fitto reticolo di gallerie collegate fra loro che percorrevano tutta la città. Alcune erano state utilizzate già dagli antichi cristiani per sfuggire alle persecuzioni, altre si erano formate a seguito delle operazioni di scavo ed estrazione del tufo utilizzato per la costruzione delle abitazioni, altre ancora costituivano una parte dell'acquedotto borbonico ${ }^{8}$. L'allestimento dei rifugi pubblici rappresentava una forte leva di propaganda del Pnf locale, il cui primario obiettivo era quello di presentare alla popolazione una situazione ben pianificata e a prova di pericolo. Tuttavia il loro definitivo completamento non procedeva nei tempi previsti, tanto che il rischio di sovraffollamento nel centro storico veniva segnalato al podestà Orgera già nel $1940^{\circ}$. Non mancarono episodi tragici, dove si contarono innumerevoli morti per soffocamento a seguito del massiccio riversamento nei rifugi delle persone in cerca di una via di fuga dai bombardamenti. Nel quartiere Montecalvario si registrarono violenti scontri fra la popolazione che, in preda al panico, si riversò in gran numero nei pochi ingressi previsti dal ricovero $^{10}$.

Il crescente disagio della popolazione trova numerosi riscontri nella documentazione prefettizia, all'interno della quale non mancano lamentele anonime nei confronti del servizio di protezione antiaerea, giudicato inadeguato e inefficiente. Al centro delle denunce più veementi vi era in primo luogo l'estrema vulnerabilità della frontiera aerea di Napoli. In occasione dei bombardamenti di ottobre gli informatori riportavano che, nonostante la risposta della contraerea, i bombardieri nemici avevano sostato sui cieli della città per cinque ore, sganciando indisturbati centinaia di bombe contro gli stabilimenti industriali e la ferrovia ${ }^{11}$.

Con l'ingresso in guerra degli Stati Uniti si aprì una nuova campagna di bombardamenti che avrebbe arrecato a Napoli ulteriori danni e vittime civili: le sorti della città ancora una volta si legarono indissolubilmente a quelle dell'intero conflitto. L'Italia, dopo le sconfitte riportate sul fronte africano, costituiva in modo ancor più lampante l'anello debole da colpire. Fiaccare il morale della popolazione con continui attacchi aerei per costringerla alla resa divenne l'obiettivo essenziale. Il 4 dicembre 1942 gli americani irruppero sui cieli di Napoli, con un primo bombardamento diurno dagli effetti devastanti. I bombardieri presero di mira il porto, per poi ripiegare sui quartieri adiacenti e l'area del centro storico. Il Palazzo delle Poste venne colpito da più ordigni, che causarono al suo interno numerose vittime. Nella adiacente via Monteoliveto

\footnotetext{
${ }^{8}$ GRIBAUDI, Gabriella, «L'impatto della guerra aerea», cit., p. 58.

${ }^{9}$ CHIANESE, Gloria, «Napoli nella seconda guerra mondiale», in Italia contemporanea, 195, 1994, pp. 343-362, p. 354.

${ }^{10}$ ASNa, Prefettura, Gabinetto, secondo versamento, b. 1222/1, Disciplina di guerra. Bombardamenti (1941-1942), Comunicato della Prefettura di Napoli, 23 ottobre 1941. Vedi anche PANE, Andrea, op. cit., p. 78 e GRIBAUDI, Gabriella, «L'impatto della guerra aerea», cit., p. 61.

${ }^{11}$ ASNa, Prefettura, Gabinetto, secondo versamento, b. 1222/1, Disciplina di guerra. Bombardamenti (1941-1942), informativa anonima, 23 ottobre 1941.
} 
furono colpite due vetture tramviarie colme di passeggeri ${ }^{12}$. Si verificarono numerosi crolli degli edifici affacciati lungo la strada, nelle vicinanze degli uffici postali messi sotto tiro non venne risparmiata la Chiesa di San Diego all'Ospedaletto, che riportò danni consistenti alla facciata e alla navata centrale ${ }^{13}$. Anche in quell'occasione si registrarono morti per soffocamento nel ricovero di via Porta San Gennaro e feriti in quello vicino di via San Paolo ${ }^{14}$. Le immagini dei corpi straziati fra cumuli indistinti di macerie e lamiere definirono i comuni contorni delle memorie cittadine di quegli anni, germogliate lungo i solchi di un'anomala temporaneità quotidiana, scandita dalle bombe e dalla successiva raccolta dei suoi tragici frutti ${ }^{15}$.

Con il 1943 si aprì l'annus horribilis per la popolazione napoletana, tenuta ormai sotto scacco dalla recrudescenza delle operazioni belliche. Il quartiere Montecalvario venne nuovamente colpito insieme all'adiacente zona di San Giuseppe durante le incursioni aeree del 1 e 5 marzo, che provocarono danni alla Chiesa di San Giacomo degli Spagnoli e crolli con vittime degli edifici in via Pasquale Scura, piazzetta Montesanto e via Pignasecca ${ }^{16}$. Il 28 marzo il misterioso incendio della Caterina Costa e del suo cospicuo carico di munizioni e carburante diretti in Africa paralizzò ulteriormente il porto, che i continui raid avevano ridotto alla stregua di una tomba per navi. La violenta esplosione, avvertita anche oltre città, aggravò il computo delle distruzioni registrate nel centro urbano a seguito della seconda campagna di bombardamenti ${ }^{17}$. Dall'altra parte, riprendeva con maggiore intensità la parallela guerra psicologica, per incutere maggior timore nella popolazione e privare definitivamente il nemico della necessaria coesione interna. Nel maggio del 1943 la RAF distribuiva fra i cieli di Napoli il seguente monito:

Hitler e Mussolini hanno condannato l'Italia a diventare la terra di nessuno. Terra di nessuno: con questo nome gli strateghi definiscono quel settore desolato, che si comprende fra due opposti fronti di combattimento. Con la liquidazione della campagna d'Africa, il posto dell'Italia, nella strategia dell'Asse è quello di un cuscinetto o paravento lungo il quale lo Stato Maggiore tedesco spera di rallentare la marcia delle Nazioni Unite [...] Se noi vi diciamo che l'Italia diventerà terra di nessuno, vi parliamo sul serio; il vostro paese sarà esposto al bombardamento, al mitragliamento, alla disorganizzazione più completa; innumerevoli case finiranno in fiamme, per città e campagne si accumuleranno cadaveri. Freddo d'inverno, infezioni d'estate, sgomento, fame si moltiplicheranno ${ }^{18}$.

\footnotetext{
${ }^{12}$ GRIBAUDI, Gabriella, «L'impatto della guerra aerea», cit., p. 42.

${ }^{13}$ VILLARI, Sergio, RUSSO, Valentina, VASSALLO, Emanuela, Il regno del cielo non è più venuto. Bombardamenti aerei su Napoli 1940-1944, Napoli, Giannini, 2005, pp. 137-139.

${ }^{14}$ GRIBAUDI, Gabriella, «L'impatto della guerra aerea», cit., p. 43.

${ }^{15}$ STEFANILE, Aldo, I cento bombardamenti di Napoli. I giorni delle AM Lire, Napoli, Marotta, 1968, p. 5.

${ }^{16}$ PANE, Andrea, op. cit., p. 79.

${ }^{17}$ PAPA, Antonio, Napoli: il trauma della liberazione, in PLACANICA, Augusto (a cura di), 1944 Salerno capitale: istituzioni e società, Napoli, Edizioni Scientifiche Italiane, 1986, p. 410.

${ }^{18}$ ASNa, Prefettura, Gabinetto, secondo versamento, b. 1224/1, Disciplina di guerra. Bombardamenti (1942-1943),
} 
Il linguaggio crudo ed esplicito mirava a colpire i civili come un pugno nello stomaco, ma l'intenzione di attaccare ovunque e indiscriminatamente fin dentro le loro abitazioni aveva da tempo preso forma. La strategia di moral bombing, laddove continuava a essere nascosta o minimizzata nei discorsi ufficiali, si presentava con estrema chiarezza per la popolazione soggetta ai bombardamenti. Il 25 luglio Mussolini era stato destituito dal Gran Consiglio fascista, tuttavia la volontà del nuovo governo, presieduto dal generale Badoglio, di proseguire la guerra sembrava immutata $^{19}$. La reazione anglo-americana non si fece attendere: nel mese di agosto vennero progettati nuovi, devastanti raid nelle maggiori città italiane. Napoli fu colpita il 4 agosto: nel terribile bombardamento diurno a tappeto bruciò la Chiesa di Santa Chiara, un complesso architettonico dal valore artistico inestimabile nonché di grande importanza sentimentale per i partenopei. L'episodio trovò ampio spazio anche nella propaganda dei cinegiornali di guerra:

Ecco gli obiettivi del nemico nelle nostre città: la monumentale Chiesa di Santa Chiara, dal Trecento, il tempo in cui venne edificata, recava in armoniose forme d'arte l'impronta di ogni secolo della storia napoletana. Oggi, dopo il novantaseiesimo bombardamento della città, tanta bellezza, tanta somma di memorie sono distrutte. Aspetti dolorosi e significativi dello scempio nemico ${ }^{20}$.

Con l'ingresso delle truppe anglo-americane in città il $1^{\circ}$ ottobre 1943 e l'insediamento del nuovo regime di occupazione, ai raid alleati si sostituirono quelli della Luftwaffe. Le incursioni naziste posero anch'esse nel mirino il centro cittadino, colpendo simbolicamente in vico Carrozzieri il giorno stesso dell'arrivo delle nuove forze occupanti ${ }^{21}$. Al terrore dei nuovi raid si aggiunse quello delle mine, che i tedeschi avevano disseminato per la città in concomitanza con la loro ritirata. L'esplosione nel Palazzo delle Poste del 7 ottobre, a seguito dell'innesco di un ordigno ritardante, causò oltre cinquanta morti nonché un ulteriore shock per la popolazione stremata dal conflitto ${ }^{22}$. Il $1^{\circ}$ novembre fu la volta dei Quartieri Spagnoli, dove si verificarono

foglio volante della RAF, maggio 1943. Vedi anche GRIBAUDI, Gabriella, «L'impatto della guerra aerea», cit., pp. 45-46.

${ }^{19}$ BALDOLI, Claudia, «I bombardamenti sull'Italia nella Seconda Guerra Mondiale. Strategia anglo-americana e propaganda rivolta alla popolazione civile», in Deportate, esuli, profughe. Rivista telematica di studi sulla memoria femminile, 13-14, 2010, pp. 34-49, p. 46.

${ }^{20}$ Archivio Istituto Luce (d'ora innanzi AIL), Giornale Luce C0369/3, L'offesa nemica su Napoli. Aspetti dolorosi e significativi dello scempio nemico, 14 agosto 1943. I materiali audiovisivi impiegati per questo articolo sono consultabili al seguente URL: < http://www.archivioluce.com/archivio/ > [consultato il 30 settembre 2016].

${ }^{21}$ STEFANILE, Aldo, op. cit., p. 170.

${ }^{22}$ Ibidem, p. 183. 
crolli in vico Tofa e in via Gesù e Maria, ingenti danni e vittime anche fra militari alleati in vico Canale, gradini San Matteo e vico Lungo Montecalvario ${ }^{23}$.

I bombardamenti tedeschi si susseguirono fino alla notte tra il 14 e 15 marzo 1944 che interessò nuovamente la zona portuale e i quartieri Montecalvario e San Giuseppe, dove venne colpito il complesso conventuale di Monteoliveto, al cui interno alloggiava la caserma dei Carabinieri Pastrengo ${ }^{24}$. Gravemente danneggiata fu la vicina Chiesa di Sant'Anna dei Lombardi: l'incursione provocò il crollo dell'intera facciata e la parziale distruzione delle cappelle ${ }^{25}$. Nel diario del soldato inglese Norman Lewis, testimone d'eccezione dei convulsi eventi in virtù del suo impiego nelle fila della Field Security Police, è possibile rintracciare alcuni cenni ai drammatici effetti dell'incursione:

Un brutto bombardamento stanotte. Come sempre, molte vittime fra i civili nella zona densamente popolata del porto. Stamattina mi ci hanno mandato a verificare le notizie pervenute di scene di panico e di folle terrorizzate che correvano per le strade gridando: "Vogliamo la pace!" e "Via tutti i soldati!". A Santa Lucia [...] ho visto una scena straziante. Lungo la strada erano stati distesi uno accanto all'altro alcuni bambini estratti dalle macerie di un edificio bombardato. A quelli non sfigurati avevano scoperto il volto, ed alcuni erano state deposte fra le braccia delle bambole nuove di zecca che li avrebbero accompagnati nell'altro mondo. [...] Un uomo si era arrampicato sulle macerie; parlava dentro a un buco, nel punto in cui credeva che il suo bambino fosse rimasto intrappolato sotto centinaia di tonnellate di detriti, e lo implorava di non morire prima che lo liberassero. "Resisti, figlio mio. Ancora un poco. Tra un minuto ti tiriamo fuori. Non morire, ti prego". I tedeschi, con questi bombardamenti indiscriminati, uccidono solo i poveracci - come abbiamo fatto anche noi, del resto ${ }^{26}$.

Al di là dei dattilografici computi delle perdite umane e materiali che affollano la documentazione d'archivio, le fonti memorialistiche hanno fornito un ulteriore contribuito per l'arricchimento del racconto storico di quegli anni cruciali e rovinosi al tempo stesso. La pioggia di ordigni che squarcia il cielo ne costituisce un topos ricorrente, sia che la rielaborazione del vissuto bellico abbia preso forma nella pietra delle edicole votive - una fra queste, quella eretta nel Rione Stella per commemorare le vittime dell'incursione del 4 agosto 1943 -, che nelle filastrocche popolari sulla guerra pervenuteci, la più nota fra queste: "Apparecchio 'e'mericane volta 'e bombe e se 'nne va / se 'nne va int'a cappuccia / apparecchio 'e'mericane volta 'e bombe e

\footnotetext{
${ }^{23}$ GRIBAUDI, Gabriella, Guerra totale, cit., p. 160.

${ }^{24}$ PANE, Andrea, op. cit., p. 83.

${ }^{25}$ VILLARI, Sergio, RUSSO, Valentina, VASSALLO, Emanuela, op. cit., pp. 80-81.

${ }^{26}$ LEWIS, Norman, Napoli '44, Milano, Adelphi, 1993, pp. 119-120.
} 
se'nne va / e se "nne va a ret 'o pizzo / e se ne fa "na bella pizza»" ${ }^{27}$. Di contro, la percezione popolare dominante sulla guerra aerea ha interessato maggiormente il recente dibattito storiografico, senza che siano state fornite a riguardo risposte univoche. Nonostante il dominio dell'uomo sulla tecnica si fosse sviluppato nella direzione di un uso ancor più cinico e spregiudicato degli strumenti di distruzione, l'impersonalità del mezzo, data principalmente dall'impossibilità di scorgere il volto dei piloti nemici, ne facilitò le strategie di legittimazione, anche a guerra ultimata. Il sentimento di impotenza nei confronti dei bombardamenti si sarebbe sviluppato così nella direzione di un generale atteggiamento fatalistico e di un'accettazione passiva della morte. Ciò può in parte spiegare l'involontaria introiezione, da parte degli sconfitti, delle modalità di discorso e dei punti di vista delle potenze vincitrici. Anche l'analisi della corrispondenza censurata dagli uffici militari $^{28}$ non fornisce prove di una maggiore consapevolezza popolare riguardo la natura terroristica dei bombardamenti a tappeto, che la stessa macchina propagandistica alleata fu costretta ad ammettere, seppur giustificandola nel quadro della superiore necessità bellica:

Modern war is cruel in its unicity has been cruel to Naples. Long before the battle on the city itself right to the day of the El Alamin murder allies bombers hammered military objectives in Naples with devasting effects. They bombed the harbour until the piers were shambles and the roads itself where choked with samples' ships. They bombed the railway station [...] They bombed everything to unable the enemy to carry on the war $^{29}$.

\section{Una difficile ricostruzione}

From the beach of Salerno the Allies blasted on Naples. The Germans couldn't do with the city so they did all they could to get it unused. Then they pulled out. When the Nazis were gone, the people of Naples saw what the atrocity of the war had done. They were let with no power, no gas, no transport, no food, no water and ultimately no homes ${ }^{30}$.

\footnotetext{
${ }^{27}$ «Aereo americano sgancia la bomba e se ne va / ritorna alla base [nota personale dell'autore] / aereo americano sgancia la bomba e se ne va / e se ne va dietro l'angolo / e lo riduce come una bella pizza» [NdT]. Vedi anche CHIANESE, Gloria, Quando uscimmo dai rifugi. Il Mezzogiorno fra guerra e dopoguerra, Roma, Carocci, 2004, p. 48.

${ }^{28}$ Il controllo della corrispondenza era di competenza dell'Ispettorato censura militare - Ufficio informazioni, a sua volta dipendente dal Ministero della Guerra. La censura era orientata a scopi militari e doveva rispettare, nei limiti delle possibilità, il principio del segreto epistolare (Premessa delle N.P.C.G., pubblicazione 1333 S.E., agosto 1944). Le lettere censurate impiegate in questo articolo sono contenute in Archivio Centrale dello Stato (d'ora innanzi ACS), Presidenza del Consiglio dei Ministri, Gabinetto, 19441947, b. 1.2.2., f. 14884, Censura postale e Ministero dell'Interno, Gabinetto, 1944-1946, b. 15, f. 1119, Censura militare. Relazioni; Censura postale. Varie.

${ }^{29}$ AIL, Combat film RW336, Naples is a battlefield. A British Ministry of Information film, The British Army Film Unit and The Royal Air Force Film Production Unit, 1944.

${ }^{30}$ Ibidem.
} 
Una volta compiuto il loro ingresso in città, agli occhi degli eserciti alleati si materializzò uno scenario desolante e di inaudita gravità che andava necessariamente affrontato. Napoli si presentava come un lugubre campo di battaglia, fatto di edifici scheletriti e pericolanti e privo dei servizi più elementari. Le testimonianze del tempo parlano di gruppi di persone che si aggiravano fameliche e impaurite tra le macerie, all'affannosa ricerca di un qualsiasi oggetto utile ai fini di scambio, nonché di lunghissime e penose file presso le poche fontane integre per attingere acqua $^{31}$. Secondo il racconto di Lewis, a causa dell'assoluta mancanza di cibo, molti bambini ricorsero all'estrazione delle patelle dal molo di Santa Lucia. Queste ultime potevano essere vendute ai bordi della strada, secondo il primo principio di sopravvivenza dettato dall'esperienza bellica che «nulla, assolutamente nulla di ciò che l'apparato digerente umano è in grado di assimilare va sprecato ${ }^{32}$. Gli effetti della guerra dei cieli, che l'occhio della cinepresa all'interno dei bombardieri alleati soleva celare in una microscopica coltre di fumo ${ }^{33}$, si manifestavano ora sulla terra in tutte le sue fattezze più dure.

I bombardamenti avevano acuito in particolar modo il problema abitativo, riducendo allo stato di senzatetto, sinistrati o sfollati un numero difficilmente quantificabile di abitanti dei quartieri più colpiti, specie nel centro storico, di modeste se non misere condizioni economiche ${ }^{34}$. La situazione edilizia a Napoli presentava ab origine numerose criticità, in buona parte riconducibili al processo di intensa urbanizzazione che aveva investito a più riprese il capoluogo. Guido Milone, ingegnere e amministratore delegato della Società per il Risanamento di Napoli, nel tentativo di delineare i caratteri di un nuovo modus operandi in materia di riqualificazione urbana, denominò un paragrafo del suo opuscolo divulgativo Per la ricostruzione di Napoli con la formula esemplificativa "Sovraffollamento e disordine edilizio". La causa principale dell'emergenza abitativa andava in primo luogo ricercata nell'eccessivo sovraffollamento, che avrebbe determinato nel corso dei secoli «un peggioramento della fisionomia della città e delle sue condizioni igieniche». In mancanza di un piano razionale di ampliamento del tessuto urbano, per rispondere ai bisogni della popolazione in continuo aumento «si sopraelevarono per due o tre piani gli edifici esistenti, si costruirono abitazioni persino sulle chiese, e si riempirono con nuove fabbriche i caratteristici cortili e giardini privati che costituivano i polmoni degli antichi

\footnotetext{
${ }^{31}$ STEFANILE, Aldo, op. cit., p. 171.

${ }^{32}$ LEWIS, Norman, op. cit., p. 37.

${ }^{33}$ AIL, Combat Film RW172, Bombardamenti alleati sull'Italia, 29 agosto 1943.

${ }^{34}$ Nel 1948, l'Ufficio Statistica del Comune di Napoli aveva rilevato nei quartieri Mercato, Poggioreale e Pendino una percentuale di vani completamente distrutti o danneggiati pari rispettivamente al 90,24, al $57,49 \mathrm{e}$ al $46,04 \%$. Ancora oggi non è possibile indicare una cifra esatta del numero degli sfollati allontanatisi dal capoluogo a causa dei bombardamenti. In mancanza di un efficace piano di evacuazione dalle grandi città, lo sfollamento da Napoli interessò principalmente coloro che riuscirono, non senza difficoltà, a trovare un punto d'appoggio nelle campagne circostanti. Vedi anche DE MARCO, Paolo, Polvere di piselli. La vita quotidiana a Napoli durante l'occupazione alleata (1943-1944), Napoli, Liguori Editore, 1996, pp. 104, 170.
} 
quartieri». In aggiunta all'angusta e disordinata rete stradale sorta intorno al nucleo dell'antica città greca, che frustrava ogni possibilità di un coerente sviluppo edilizio,

le condizioni generali di depressione delle industrie e dei commerci più tradizionali di Napoli, la mancanza di scuole artigiane e le inadatte sedi delle scuole di istruzione pubblica, per la maggior parte allogate in antichi conventi o in vecchi locali di abitazione, malsani e del tutto insufficienti ai bisogni della gioventù; il difettoso funzionamento delle fognature che inquina giornalmente il litorale urbano e provoca il diffondersi della febbre tifoide; insieme con altri fattori negativi concomitanti, avevano determinato, già prima dell'attuale guerra, uno stato eccezionale che trova conferma nelle condizioni di miseria morale e fisica del popolo ${ }^{35}$.

In un successivo paragrafo, denominato "Case per il popolo", si affrontava il tema delle «case economiche», definite come «un punto vitale al quale bisognerà provvedere per il miglioramento igienico e sociale del popolo». La necessità di incentivare la costruzione di abitazioni popolari veniva posta in diretta correlazione, non senza un velo di paternalismo, con l'auspicata elevazione morale e materiale della "plebe napoletana". Il possesso di una casa, il più possibile incasellabile all'interno di riconosciuti parametri di decoro, secondo le intenzioni del pianificatore le sarebbe valso da canale privilegiato per il proprio riscatto e il conseguente reinserimento nella società nelle vesti di umili, ma onesti lavoratori.

Lo Stato vedrà nell'abitazione sana e nella scuola le armi migliori per combattere l'alta mortalità e morbilità ed il progressivo aggravarsi dell'indigenza e delle tendenze antisociali che purtroppo si osservano in questa disgraziata metropoli. Anche perché il problema degli alloggi popolari non deve essere limitato alla classe degli operai i quali, attingendo dal lavoro modesti ma pur sicuri mezzi di sussistenza ed abitudini di civile convivenza, possono meglio reagire contro le malefiche influenze di una casa malsana. A Napoli esiste disgraziatamente una plebe assai numerosa, sproporzionata al resto della cittadinanza, che vive miseramente, senza una vera arte o mestiere, ma piuttosto di espedienti, di vagabondaggio o di elemosina. È evidente che il miglioramento dell'abitazione costituisce la condizione pregiudiziale per l'incivilimento delle masse, ed è uno dei cardini per la grande riforma che dovrà trasformare il vagabondo in onesto operaio e dare al popolo la coscienza di una maggiore dignità personale ${ }^{36}$.

L'esigenza di destinare case minime, ovvero a basso costo, per uso popolare si era presentata già nel 1941. Gli alloggi, destinati alle fasce più indigenti della popolazione, dovevano fornire una prima soluzione ai problemi di migliaia di sinistrati che avevano visto le loro abitazioni

\footnotetext{
${ }^{35}$ MILONE, Guido, Per la ricostruzione di Napoli, Napoli, Richter, 1944, pp. 11-14, pubblicazione a cura e spesa del Banco di Napoli.

${ }^{36}$ Ibidem, pp. 31-34.
} 
gravemente danneggiate o distrutte dalle incursioni aeree. Nel gennaio 1941 venne autorizzata la costruzione, a spese dello Stato, di stabili di tipo popolarissimo per il ricovero delle famiglie senzatetto. Lo schema di convenzione predisposto dall'Amministrazione per i lavori pubblici disciplinava le modalità di gestione di tali alloggi, prevedendo la consegna da parte dello Stato agli Enti Comunali di Assistenza e l'assunzione degli oneri di amministrazione e manutenzione da parte degli Istituti provinciali per le case popolari ${ }^{37}$.

L'allora Consorzio Nazionale degli Istituti fascisti autonomi per le case popolari, con circolare del 15 febbraio 1942, mise a punto la convenzione con i periferici Istituti fascisti provinciali per le case popolari e gli enti assistenziali loro associati. Nel testo si leggeva che era «stata riconosciuta la necessità di una collaborazione permanente fra gli istituti predetti e gli enti comunali di assistenza, per realizzare un adeguato programma di costruzione di case minime, da destinare alle famiglie indigenti». Di conseguenza, «in base alle disposizioni impartite dal Ministero dell'Interno e dal Consorzio, all'attuazione dei programmi delle singole provincie, affidata agli Istituti fascisti, devono concorrere, nella misura di un terzo del costo delle costruzioni, gli ECA interessati, assumendosi gli Istituti gli oneri del finanziamento della residua spesa ${ }^{38}$. Tuttavia, man mano che procedeva il conflitto e con esso l'urgenza di ricollocare un numero sempre maggiore di sinistrati, la loro costruzione divenne sempre più frettolosa e disordinata, con ripercussioni visibili per la messa in sicurezza degli abitati. Dalla documentazione prefettizia emergono, infatti, notizie di innumerevoli crolli causati delle pessime condizioni strutturali degli edifici che provocarono un vivo malcontento popolare ${ }^{39}$.

I problemi strutturali delle case minime si protrarranno anche nel dopoguerra. Una nota dell'Istituto per le case popolari della provincia di Napoli (l'organo che sostituì ed ereditò le competenze di materia dell'Istituto fascista provinciale per le case popolari) del luglio 1944 declinava ogni responsabilità inerente i crolli futuri, in quanto gli obblighi di manutenzione degli edifici da parte di quest'ultimo non coprivano il lavoro di rafforzamento dei solai, ormai gravemente dissestati. In mancanza di provvedimenti a riguardo da parte del Genio Civile, le case dovevano essere dichiarate inagibili. Il prefetto, messo al corrente della situazione, nel mese di marzo aveva ordinato l'affissione di un avviso nei rioni in oggetto, dove si allertavano gli inquilini della pericolosità degli abitati ${ }^{40}$.

Nell'ottobre dello stesso anno, il Genio Civile, in una nota rivolta al prefetto di Napoli, dichiarava che da un'ispezione eseguita dal suo ufficio risultava che diciotto alloggi del blocco

\footnotetext{
${ }^{37}$ ASNa, Prefettura, Gabinetto, secondo versamento, b. 1231/1, Disciplina di guerra. Bombardamenti (1943-1946), Ministero del Tesoro - Terza Divisione al Ministero dei Lavori Pubblici e all'Istituto per le case popolari di Napoli, n. prot. 136575/135420, Roma 27 novembre 1945.

${ }^{38}$ Ibidem, Consorzio nazionale fra gli Istituti fascisti autonomi per le case popolari a tutti gli Istituti fascisti provinciali per le case popolari e a tutti gli altri enti associati, circ. n. 185, Roma 15 febbraio 1942.

${ }^{39}$ Ibidem, Case per i sinistrati dalle incursioni nemiche, Napoli 13 gennaio 1942.

${ }^{40}$ Ibidem, Istituto per le case popolari al Comune di Napoli, doc. n. 2414, Napoli 14 luglio 1944.
} 
delle case minime di Miano necessitavano di un sollecito intervento di ristrutturazione. Si aggiungeva inoltre che, "per l'attuale e assoluta mancanza di abitazioni o ricoveri disponibili», si sarebbe proceduto a sgombrare i soli ambienti che necessitavano urgenti lavori di riparazione, richiedendo allo stesso tempo agli inquilini coinvolti da tali misure di trasferirsi momentaneamente «dalla famiglia dell'abitazione adiacente o da altra dello stesso rione» ${ }^{41}$.

Non mancarono tuttavia le difficoltà: nei casi più difficili, il Genio Civile richiese al prefetto l'invio delle autorità di pubblica sicurezza nelle case minime di via Montedonzelli all'Arenella e in quelle di Miano, in quanto due inquilini alloggiati in quelle abitazioni si opponevano ai lavori di ristrutturazione dei rispettivi solai ${ }^{42}$. Ulteriori lamentele per le pessime condizioni delle case minime vennero sollevate dagli stessi abitanti, che denunciavano il più delle volte l'estrema lentezza dei lavori di rifinitura dei soffitti interessati, nonché la fitta presenza di insetti velenosi all'interno degli abitacoli ${ }^{43}$.

Per offrire una risposta unitaria all'emergenza sinistrati, venne messa in moto anche la rete di primo soccorso. L'Ufficio speciale per i danneggiati di guerra dell'ECA garantiva una serie di sussidi, ripartiti in questa misura: per la casa interamente crollata 1500 lire al capofamiglia e 500 lire a ogni componente della famiglia; per la casa parzialmente crollata 1200 lire al capofamiglia e 400 lire a ogni componente; per la casa dichiarata inabitabile 900 lire al capofamiglia e 300 lire a ogni componente. Le principali strutture adibite per il ricovero dei nuclei familiari sinistrati erano il Reale Albergo dei Poveri, il Piccolo Cottolengo - che furono costretti a sospendere per un periodo limitato di tempo il servizio, a causa delle incursioni belliche del settembre 1943 -, e il Vittorio Emanuele III, quest'ultimo con una capacità ricettiva di 500 persone. L'ECA garantiva al contempo la copertura delle rette giornaliere richieste da questi ultimi. Fino alla completa riassunzione del servizio di assistenza sinistrati da parte dell'ente, i disciolti gruppi rionali fascisti gestirono direttamente la somministrazione di vitto e alloggio, previo rimborso spese.

Parallelamente all'assegnazione delle 700 case minime nelle zone Arenella, Scudillo a Capodimonte, Miano, Barra e San Giovanni a Teduccio, era previsto un servizio di distribuzione degli indumenti presso i magazzini presenti nei maggiori centri di ricovero, i cui stessi locali non vennero risparmiati dalle incursioni aeree. Nella competenza dell'Ufficio danneggiati di guerra rientrava inoltre l'assistenza agli sfollati, elargita anche essa sotto forma di sussidio, nella cifra di 100, 75 o 50 lire a persona a seconda della distanza del luogo di trasferimento, nonché di fruizione di biglietti gratuiti per la ferrovia. La filiale dell'assistenza copriva infine le vedove e le madri dei caduti in guerra, con un sussidio mensile che si aggirava fra le 75 e le 100 lire; i familiari più

\footnotetext{
${ }^{41}$ Ibidem, Ufficio del Genio Civile al prefetto di Napoli, doc. n. 17961, Napoli 7 ottobre 1944.

${ }^{42}$ Ibidem, Ufficio del Genio Civile al prefetto di Napoli, doc. n. 30961 e n. 30963, Napoli 17 settembre 1945.

${ }^{43}$ Ibidem, esposto al ministro dei Lavori Pubblici, al sindaco e al prefetto di Napoli, doc. n. 7279, Napoli 9 maggio 1946.
} 
indigenti dei richiamati al fronte; i militari in convalescenza per cause di servizio con un sussidio di 100 lire mensili e la somministrazione gratuita di medicinali; e categorie speciali di disoccupati per cause di guerra. Gli edifici scolastici, adibiti a centri di ricovero dalla Federazione provinciale fascista, continuarono a servire per tale scopo anche dopo il suo scioglimento. Le sedi dei soppressi gruppi rionali fascisti vennero utilizzate in seguito come locali decentrati per il pagamento dei sussidi ai sinistrati ${ }^{44}$.

Il riconoscimento dei danni di guerra, con corresponsione del relativo indennizzo agli aventi diritto, richiedeva l'adempimento di un'articolata procedura. Secondo le disposizioni emanate dal Ministero del Tesoro per snellire le procedure di accertamento, le Intendenze di finanza dovevano cooperare, oltre che con la Guardia di finanza, anche con le questure e le forze dell'ordine. Era necessario prendere informazioni su: le condizioni economiche passate e attuali del richiedente; le condizioni sociali, il tenore di vita; sull'attività lavorativa del capofamiglia; la data dell'evento bellico che aveva originato il sinistro e gli eventuali sfollamenti e trasferimenti di mobilio prima e dopo il sinistro. Era inoltre indicato per le autorità competenti un sopralluogo nella località del sinistro, per descrivere i danni riportati dallo stabile, e infine nell'attuale dimora del sinistrato. Bisognava inoltre distinguere i danni accertati, per i quali erano state esibite prove certe, da quelli non accertati ed estendere le indagini del caso nelle località dove il richiedente era eventualmente sfollato ${ }^{45}$.

Non mancarono casi in cui la procedura presentava lacune e inadempienze da parte degli esaminatori, e di conseguenza venne rilevato un cospicuo numero di pratiche falsificate, atte ad ottenere un risarcimento non dovuto ${ }^{46}$. Una nota a firma del questore aggiungeva, inoltre, che la stessa Intendenza di finanza aveva rilevato una notevole mole di risarcimenti per danni di guerra denunciati in case abbandonate prima dell'avvenuto sinistro. Le richieste erano pervenute in

\footnotetext{
${ }^{44}$ Ibidem, Ente Comunale di Assistenza di Napoli - Ufficio speciale danneggiati di guerra, settembre 1943. Gli edifici scolastici adibiti a centri di ricovero dalla disciolta Federazione provinciale fascista erano: scuola Duse di Porta Posillipo, scuola media in via Campi Flegrei, scuola Oriani in San Pietro a Patierno, scuola in via Duca d'Aosta a Pianura, scuola Aganoor in Corso Napoli a Marianella, asilo in via Vittorio Emanuele a Piscinola, scuola materna a Chiaiano, scuola Toti a Ponticelli (capienza di 300 posti), scuola Corridoni a Barra (capienza di 300 posti), scuola Russo in via Santa Margherita a Fonseca (capienza di 150 posti), scuola Bovio in via San Giovanni a Carbonara, scuola Petrarca alla Salute (capienza di 150 posti), scuola Mastriani in via Ferriera a Poggioreale, scuola Taddei a Miano, asilo Pascoli a Miliano, scuola a Piazza Mondragone, Liceo Umberto I (capienza di 520 posti), scuola media in Santa Maria in Portico (capienza di 520 posti), scuola De Amicis in via Santa Teresa a Chiaia. Le sedi dei disciolti gruppi rionali fascisti, adibite al pagamento dei sussidi per i sinistrati, erano: "Baracca" al rione Santa Croce all'Arenella, "Luporini" in Piazza Torretta e "Aurelio Padovani" in Piazza Padovani. Nel documento si fa cenno alla volontà di ampliare tale servizio, usufruendo dei locali "Buonservizi" in via Vergini, "Nardini" a Secondigliano e "Neri" a San Giovanni a Teduccio.

${ }^{45} \mathrm{Ibidem}$, comunicato della Questura di Napoli alle forze di polizia, al prefetto e all'Intendenza di finanza di Napoli, doc. n. 107144, Napoli 1 maggio 1945.

${ }^{46}$ ASNa, Questura, Gabinetto, Massime, b. 126, f. 2768, Danni di guerra, informativa urgente della Decima legione territoriale della Guardia di finanza "Vesuvio" di Napoli all'Intendenza di finanza, alla Questura e al Comando Compagnia interna dei carabinieri di Napoli, n. prot. 1038, Napoli 24 maggio 1946.
} 
particolar modo per le abitazioni site in quartieri quasi del tutto distrutti, come Poggioreale. Le segnalazioni da parte dell'Intendenza inducevano il questore a ritenere che le procedure di accertamento dei danni di guerra non venivano condotte «con quella diligenza necessaria e indispensabile per garantire gli interessi dell'erario». Precisava inoltre che nell'esame delle pratiche occorreva accertare in primo luogo che la casa era occupata dal richiedente all'atto del sinistro, anche con l'ausilio di prove indirette richiedendo l'esibizione delle bollette dell'energia elettrica o del gas, riferibile al mese precedente all'incursione. Era necessario stabilire che gli altri componenti della famiglia non avessero presentato ulteriori domande di risarcimento e, dunque, nei rapporti bisognava indicare anche i nominativi di tutti gli abitanti della casa all'atto del sinistro. Per il computo dei danni, era necessario interrogare nei limiti delle possibilità anche i vicini dei richiedenti. Qualora questi ultimi si fossero trasferiti in un'altra abitazione a seguito dei bombardamenti, occorreva richiedere la collaborazione del commissariato di pubblica sicurezza nella cui giurisdizione si erano stabiliti, per assicurarsi che il trasferimento fosse avvenuto in una fase precedente o successiva al sinistro ${ }^{47}$.

Per poter meglio comprendere l'entità dell'emergenza abitativa, bisogna innanzitutto fare i conti con le stime dei danni arrecati dai bombardamenti. Le cifre sono discordanti: secondo un censimento angloamericano del settembre 1944, si contavano 80147 vani distrutti e Napoli aveva subito danni fra il $5 \%$ e il $10 \%$ del suo intero patrimonio abitativo ${ }^{48}$. Su richiesta del prefetto il Genio Civile aveva conteggiato per il solo capoluogo 28900 vani completamente distrutti, 61500 vani danneggiati gravemente, 143000 vani danneggiati lievemente. Per i comuni della provincia le cifre ammontavano a: 3766 vani completamente distrutti, 6765 vani danneggiati gravemente, 19968 vani danneggiati lievemente. Il totale dei danni subiti, fra capoluogo e provincia, era di: 32666 vani completamente distrutti, 68265 vani danneggiati gravemente, 162968 vani danneggiati lievemente. Secondo le ultime stime presenti nel rapporto, il numero delle persone fuggite da Napoli verso i vari comuni della provincia ammontava a 40 mila unità ${ }^{49}$.

L'entità del problema degli sfollati procedeva di pari passo con la violenza e l'ampiezza dei bombardamenti sulla città. Nell'aprile del 1943 beneficiarono del fondo speciale ECA per gli sfollati in gravose condizioni di bisogno, elargito dalla Prefettura secondo un tetto massimo di 500

\footnotetext{
${ }^{47}$ Ibidem, circolare del questore agli uffici di PS di città e provincia, n. 1017463 gab., Napoli 17 giugno 1946.

${ }^{48}$ Commissione Alleata di Controllo - Presidenza del Consiglio dei Ministri, Censimenti ed indagini per la ricostruzione industriale eseguiti nel settembre 1944, Roma, Istituto Poligrafico dello Stato, 1945, grafico 5. Vedi anche CHIANESE, Gloria, «Napoli nella seconda guerra mondiale», cit., p. 347.

${ }^{49}$ ASNa, Prefettura, Gabinetto, secondo versamento, b. 1231/1, Disciplina di guerra. Bombardamenti (1943-1946), Danni di guerra ai fabbricati privati e numero di persone rimaste senza tetto, doc. n. 35025, 10 dicembre 1945. I comuni di competenza del Genio Civile di Napoli erano: Afragola, Arzano, Bacoli, Caivano, Calvizzano, Cardito, Casalnuovo, Casola, Casoria, Castellammare di Stabia, Cercola, Giugliano, Gragnano, Ischia, Marano, Massalubrense, Monte di Procida, Mugnano, Ottaviano, Poggiomarino, Pollena Trocchia, Pomigliano d'arco, Pompei, Portici, Pozzuoli, Qualiano, Resina, Sant'Anastasia, San Giorgio a Cremano, San Giuseppe Vesuviano, Somma Vesuviana, Sorrento, Torre Annunziata, Torre del Greco, Terzigno, Villaricca.
} 
lire a persona e un minimo di 50 lire a persona, novantuno persone residenti nel capoluogo e nella provincia. Nei restanti mesi, il numero dei sussidiati subì un andamento leggermente discendente, attestandosi sulle settantasei unità a maggio; cinquantasei unità a giugno; settantaquattro unità a luglio; sessanta unità a agosto e quarantasei unità a settembre ${ }^{50}$.

Nell'ottobre 1945 il sindaco di Napoli Gennaro Fermariello comunicava alla Prefettura che nella città si registravano circa 200mila persone senza tetto ricoverate presso i familiari o alloggi di fortuna. I vani abbandonati per inagibilità si contavano nel numero di 58700 unità, mentre altri, nonostante l'inabitabilità, erano stati occupati per necessità. Si accennava alla parallela questione delle requisizioni alleate, che verrà approfondita in seguito, le quali avrebbero sottratto alla pubblica disponibilità altri 15mila vani. Si sottolineava infine una situazione preoccupante di sovraffollamento all'interno delle abitazioni, con gravi ripercussioni per l'igiene ${ }^{51}$. L'anno precedente, in un'intervista rilasciata al quotidiano «Il Risorgimento», il Commissario dell'Istituto per le case popolari della provincia di Napoli Alfredo Florio dichiarava che molti quartini a più vani, a causa della caduta dei tramezzi, «si sono trasformati in un unico ambiente, ove vivono promiscuamente intere famiglie, con quale offesa della morale e danno dell'igiene è facile immaginare $»^{52}$.

All'arrivo degli Alleati, un numero imprecisato di persone, fra le 12 e le 20mila unità, alloggiava nei malsani ricoveri, nelle stazioni della metropolitana e della funicolare ${ }^{53}$. Nel 1944, su pressione dei nuovi occupanti, la Questura di Napoli stabiliva che 11930 persone erano ancora insediate nei ricoveri, e soggette di conseguenza ad un rischio maggiore di epidemi ${ }^{54}$. Nel marzo 1944, il comune riportava che la Typhus Sub-Commission, alle strette dipendenze della Commissione di Salute Pubblica dell'Allied Control Commission (ACC), aveva censito un numero di 5705 persone distribuite su diciannove rifugi. Pur sostenendo la riduzione del loro numero a 2010 unità, dichiarava l'impossibilità di provvedere ad una sistemazione per i senzatetto, in quanto i dormitori comunali risultavano al completo. La medesima sub-commissione, al contrario, censiva per il mese di settembre un numero di 4932 persone presenti in tali rifugi, cifra che nel mese di dicembre sarebbe scesa a 2171 unità, e declinava ogni responsabilità per l'igienizzazione dei ricoveri stessi ${ }^{55}$.

La mancanza di abitazioni agibili costringeva altre persone a risiedere negli alloggi sinistrati, sebbene il pericolo rappresentato dai possibili crolli fosse elevato. La loro precaria stabilità

\footnotetext{
${ }^{50}$ ASNa, Prefettura, Gabinetto, secondo versamento, b. 1211/1, Disciplina di guerra. Sinistrati: varie (sussidi, speculazioni a danno dei sinistrati, Movimento sfollati 1943 - 1944), Fondo ECA di 10mila lire per gli sfollati.

${ }^{51}$ Ibidem, esposto del sindaco di Napoli alla Prefettura, doc. n. 718, 16 ottobre 1945.

${ }^{52}$ «Problemi del giorno. La crisi delle abitazioni e le case popolari», in Il Risorgimento, II, 229, 23 settembre 1944, p. 4.

${ }^{53}$ PAPA, Antonio, op. cit., p. 411.

${ }^{54}$ GRIBAUDI, Gabriella, Guerra totale, cit., p. 172.

${ }^{55}$ ACS, Allied Control Commission (d'ora innanzi ACC), 10260-163/57, Ricoveri, bobina 1001B, scaffale 185.
} 
costituiva un notevole problema per la pubblica incolumità, anche a seguito delle abbondati piogge che potevano provocare ulteriori cedimenti ${ }^{56}$. Ciononostante, alla scrivania del prefetto giungevano innumerevoli segnalazioni, inerenti le occupazioni di edifici sinistrati da parte di famiglie in difficoltà. Il Genio Civile rilevava una situazione particolarmente critica, sia sul versante dell'inagibilità di detti stabili, che su quello della ricollocazione degli occupanti. Per tale ragione, veniva richiesto un celere intervento da parte dello Stato per la messa in sicurezza delle abitazioni non danneggiate gravemente ${ }^{57}$.

In virtù della legge n. 938 del 9 luglio 1940, concernente gli interventi per le opere pubbliche compromesse da azioni belliche, tali oneri di spesa ricadevano in mano statale ${ }^{58}$. Tuttavia, in mancanza di un piano di ristrutturazioni edilizie ad ampio raggio, l'Allied Military Government $(\mathrm{AMG})$ si attivò in favore di un programma di riparazioni straordinario. Sull'esempio alleato, il ministro dei Lavori Pubblici Mancini autorizzò il medesimo intervento anche per le altre province che avevano subito ingenti danni di guerra ${ }^{59}$. Un numero ancora cospicuo di persone rimaneva di contro senza un tetto, tanto che il fenomeno delle occupazioni di edifici abbandonati coinvolse anche appartamenti di proprietà di istituti religiosi, i quali, essendo stati nella maggior parte dei casi gravemente danneggiati dai bombardamenti, venivano resi abitabili nei limiti del possibile dagli stessi occupanti. Come comunicavano al prefetto le Suore Salesiane di San Giovanni Bosco, tenutarie di un orfanotrofio in via Porta di Massa compromesso dalle incursioni aree del giugno 1943 ,

famiglie estranee sono penetrate nel locale sinistrato e si sono alloggiate da proprietari occupandolo e eseguendo provvisori lavori di adattamento. Interpellati, si è avuto la risposta di essere sinistrati e che non intendevano abbandonare lo stabile [...] Essi si sono violentemente rifiutati di abbandonare l'edificio, rispondendo che vi si trovavano troppo bene per lasciarlo, e anzi invitavano parenti e amici a raggiungerli, aumentando il numero degli invasori. [...] L'Orfanotrofio di orfane di guerra è da preferire all'interesse privato di poche famiglie le quali troveranno modo di alloggiarsi altrove. [...] La evidente utilità dell'opera che deve alloggiarsi nel locale renderebbe anche giustificato un provvedimento di requisizione e di sgombro del locale ${ }^{60}$.

\footnotetext{
${ }^{56}$ ASNa, Prefettura, Gabinetto, secondo versamento, b. 1211/1, Disciplina di guerra. Sinistrati: varie (sussidi, speculazioni a danno dei sinistrati, Movimento sfollati 1943 - 1944), segnalazione dei carabinieri di Napoli alla Prefettura, n. prot. 319/128, Napoli 4 novembre 1944.

${ }^{57}$ Ibidem, Ufficio del Genio Civile al prefetto di Napoli, Danni di guerra. Fabbricati sinistrati e pericolanti nella città di Napoli, doc. n. 16967, Napoli 2 gennaio 1945.

${ }_{58}$ Ibidem, Ministero dell'Interno - Direzione generale amministrazione civile ai prefetti delle provincie liberate, doc. n. 1/6121-1-10-F. div. 1, Salerno 15 giugno 1944.

${ }^{59}$ Ibidem, Ministero dei Lavori Pubblici ai prefetti dell'Italia libera, ai sindaci delle città capoluogo, agli ispettori generali e agli ingegneri capi del Genio Civile, doc. n. 24592, Salerno 30 giugno 1944.

${ }^{60}$ ASNa, Prefettura, Gabinetto, secondo versamento, b. 502/50, Orfanotrofio San Giovanni Bosco, esposto delle Suore Salesiane di San Giovanni Bosco al prefetto, Napoli 24 gennaio 1945.
} 
$\mathrm{Su}$ sollecito del prefetto, la Questura di Napoli riportava che negli stabili appartenenti all'orfanotrofio alloggiavano tre famiglie e una giovane donna dall'ottobre del 1944, in quanto le loro case erano state distrutte a seguito delle incursioni del 1 marzo e del 4 agosto 1943. Alla diffida di sgombero, gli occupanti dichiaravano che non avevano altro luogo in cui alloggiare ${ }^{61}$. La vicenda non vede un prosieguo nelle carte d'archivio, ma costituisce di per sé uno fra i tanti episodi riscontrabili nell'indagine del complesso (dis)ordine sociale postbellico, in cui il conflitto stridente fra le ragioni del diritto e quelle del bisogno non trovavano un terreno d'incontro nemmeno attraverso la mediazione delle autorità competenti.

Di considerevole entità era anche la questione degli sfratti, tanto da richiedere anch'essa il diretto intervento dell'AMG. Sempre «Il Risorgimento», utilizzato dalle autorità alleate come cassa di risonanza per la diffusione delle proprie iniziative, sosteneva che l'azione degli angloamericani avrebbe costituito un argine alle speculazioni dei proprietari ${ }^{62}$. L'annuncio di sospensione degli sfratti venne comunicato anche dal Commissario di zona, il colonnello Simson, durante una conferenza stampa in cui poneva come primo problema rilevante della città quello delle abitazioni ${ }^{63}$. Il provvedimento alleato, di contro, non pose un freno all' aumento vertiginoso dei prezzi degli immobili, che costringeva moltissime persone a sostenere spese onerose per l'affitto di alloggi spesso inadeguati e fatiscenti. È possibile farsi un'idea delle loro critiche condizioni di vita in primo luogo attraverso le richieste di sussidio indirizzate al prefetto. Le richiedenti erano spesso donne, con mariti o figli dispersi al fronte, con prole a carico e prive dei mezzi di sostentamento. Altre invece erano sinistrate prive di appoggi familiari, come viene testimoniato in una lettera datata 2 agosto 1943, due mesi prima dell'ingresso degli Alleati in città:

Residente a San Giovanni a Teduccio, sola, orfana di padre e madre, si è ritrovata povera a causa dei sinistri avvenuti nella sua abitazione. Chiede di essere aiutata perché muore di fame e non ha né il pane per potersi sostenere né i soldi sufficienti per sgombrare e affittare un'altra misera stamberga. Non ha un letto e dorme su un tavolo ${ }^{64}$.

\footnotetext{
${ }^{61}$ Ibidem, Questura di Napoli, n. prot. 103154, risposta a nota prefettizia n. 2240 del 12 febbraio 1945, Napoli 22 aprile 1945.

${ }^{62}$ «La questione degli sfratti. L'Ufficio per le pratiche fittuarie esaminerà tutte le decisioni», in Il Risorgimento, II, 85, 8 aprile 1944, p. 4.

${ }^{63}$ «Saranno emessi provvedimenti per fare in modo che la popolazione non abbia a risentire più a lungo $i$ danni della mancanza di abitazioni. A tal proposito si provvederà perché gli sfratti siano sospesi, perché gli inquilini non siano minacciati dai padroni di casa con pretese che, in questo momento, appariscano più che mai inopportune». "L'interessamento del Col. Simson per i problemi più urgenti», in Il Risorgimento, III, 4, 5 gennaio 1945, p. 4.

${ }^{64}$ ASNa, Prefettura, Gabinetto, secondo versamento, b. 1211/1, Disciplina di guerra. Sinistrati: varie (sussidi, speculazioni a danno dei sinistrati, Movimento sfollati 1943 - 1944), Richieste di sussidio alla Prefettura di Napoli.
} 
A fornire un quadro ulteriore sull'argomento è infine la corrispondenza censurata, che venne commentata dalle stesse autorità censorie nei termini inequivocabili di «triste situazione degli alloggi a Napoli»:

A Napoli il problema della casa è quasi di impossibile risoluzione. Noi viviamo in alloggi di fortuna... a nessun prezzo si trovano abitazioni disponibili, a meno che non si abbia la possibilità di pagare delle decine di migliaia di lire per le spese di ceditura [buona uscita] dell'appartamento / Per il fitto della camera la padrona vuole 1500 lire a persona ${ }^{65}$. Per ora, per mancanza di alloggi, perché vi sono molte case sinistrate, ci tocca arrangiarci in casa di zio Umberto. Se sapessi quello che si sta passando: mamma non lo credeva della crisi e miseria che esiste a Napoli. Io dormo ancora in casa dei Berletta, Vincenzino non può dormire da zio Umberto, in quel bugigattolo, sembra un sotterraneo, senza aria, senza gabinetto, umido, sempre con la luce accesa perché non vi passa uno spiraglio di luce. Mamma e Maria sono costrette ad abitarci, perché non si trova nulla. Vincenzino dorme con una coperta allo scoperto sulla terrazza dell'abitato ${ }^{66}$.

\section{I primi effetti dell'occupazione alleata}

Il regime d'occupazione alleato insediatosi nell'Italia meridionale può essere considerato come il prodotto contradditorio di un difficile compromesso, a cui dovettero necessariamente adattarsi inglesi e americani, circa la forma di controllo più adeguata da approntare per l'amministrazione di quei territori. Il dissidio di fondo, concernente la possibilità di instaurare un controllo diretto o indiretto delle aree sottoposte all'occupazione, divideva i due alleati lungo i solchi di un diverso orizzonte interpretativo degli eventi, dettato in primo luogo dalle specifiche esigenze nazionali. La proposta americana, che propendeva verso una strategia di controllo diretto, mirava di fatto ad un netto ridimensionamento della sovranità italiana per favorire un ampio margine d'azione sul territorio dei locali funzionari alleati ${ }^{67}$. Tuttavia, nel processo di definizione di un efficace modello di governo militare, gli inglesi fecero valere sull'ago della bilancia il duplice peso dei loro interessi strategici e della maturità raggiunta sul piano tattico-militare. La strategia di controllo indiretto delle retrovie, che presentava innumerevoli vantaggi strategici specie nella garanzia di law and order con il minimo dispendio di uomini, prese così il sopravvento sulle prime intenzioni

\footnotetext{
${ }^{65}$ ACS, Ministero dell'Interno, Gabinetto, 1944-1946, b. 15, f. 1119, Censura militare. Relazioni, Relazione mensile maggio 1945, lettere da Napoli.

${ }^{66}$ Ibidem, Censura postale. Varie, lettera da Napoli per New York, 28 luglio 1945. Vedi anche PORZIO, Maria, op. cit., p. 72.

${ }^{67}$ ATERRANO, Marco Maria, Mediterranean-First? La pianificazione strategica anglo-americana e le origini dell'occupazione alleata in Italia 1939-1943, Napoli, Federico II University Press, 2017, p. 186.
} 
dell'alleato d'oltreoceano, fornendo allo stesso tempo le linee guida essenziali per le successive politiche condotte nella penisola ${ }^{68}$.

Per la primaria necessità di limitare il proprio coinvolgimento nei territori occupati, si giunse dunque alla tortuosa accettazione di una coesistenza con la monarchia e il governo di Badoglio e infine al riconoscimento per il vecchio nemico dello status di Paese cobelligerante. I convulsi avvenimenti del 1943 avevano aperto tuttavia delle ferite lancinanti in seno al contesto politicosociale italiano, che misero duramente alla prova la volontà alleata di farsi carico in minima parte delle proprie responsabilità. Le devastazioni comportate da tre anni di guerra totale, l'implosione dell'apparato statale fascista a seguito dell'armistizio, la presenza dell'esercito tedesco nelle aree del centro-nord, l'incompiuto ricambio della classe dirigente e il disorientamento patito dalle masse rappresentavano i maggiori problemi di cui il debole Stato italiano insediatosi nel Meridione non era in grado di provvedere da solo. Disorganizzato al proprio interno, nonché alle prese con una complicata opera di legittimazione delle sue fondamenta istituzionali, quest'ultimo non sarebbe mai riuscito a recuperare le proprie funzioni in breve tempo e con le sue sole forze. Per sopravvivere, non poteva far altro che attendere il sostegno politico e materiale alleato. Andarono così delineandosi i primi sintomi di un rapporto di mutua dipendenza che avrebbe unito controllori e controllato, vincitori e vinto per tutta la durata dell'occupazione, segnandone irrimediabilmente l'ambiguità.

La mancanza di un forte interlocutore statale sul territorio finora conquistato condizionò in modo preponderante le strategie altalenanti degli Alleati, che propendevano, a seconda delle eventualità, sia verso la direzione del controllo indiretto che su quella del controllo diretto. In occasione dello sbarco in Sicilia, venne istituito l'Allied Military Government of Occupied Territory (AMGOT), presieduto dal generale inglese e comandante delle forze d'occupazione in Italia, Harold Rupert Alexander. Le province italiane vennero raggruppate all'interno di Regions centralizzate che seguivano l'ordine di avanzata degli eserciti alleati lungo la linea del fronte: la Region I corrispondente alla Sicilia, la Region II alla Calabria, alla Basilicata e alla Puglia, la Region III alla Campania e la Region IV alla Sardegna. Nella giurisdizione della Region III rientravano in particolare le province di Napoli, Avellino e Benevento, quella di Salerno vi avrebbe infine fatto capolino nel febbraio $1944^{69}$. Ogni provincia era affidata alla vigilanza di un Senior Civil Affairs Officer (SCAO) col compito di monitorare l'attività del prefetto e da cui dipendevano i Civil Affairs Officers (CAO) stanziati nei comuni principali. Ogni SCAO rispondeva a sua volta all'autorità del governatore regionale, il Regional Civil Affairs Officer (RCAO), il quale dirigeva la Region di appartenenza per conto del Governo militare ${ }^{70}$.

\footnotetext{
${ }^{68}$ Ibidem, pp. 183-185.

${ }^{69}$ PORZIO, Maria, op. cit., pp. 36-37.

${ }^{70}$ DE MARCO, Paolo, «Il difficile esordio del governo militare e la politica sindacale degli Alleati a Napoli», in
} 
Il Governo militare, che avrebbe assunto nell'ottobre 1943 la denominazione di AMG, esercitava la propria influenza nei territori esclusi dall'amministrazione italiana attraverso un sistema di governo parallelo, che accentrava su di sé il disciplinamento di tutti gli aspetti della vita politica, sociale e economica delle realtà locali. Come era stato stabilito ai sensi dell'art. 37 dell'Armistizio lungo, siglato fra le due controparti il 29 settembre 1943, venne istituita nel mese di novembre l'Allied Control Commission (ACC). A quest'ulteriore moloch, nato dalla sinergia burocratico-amministrativa angloamericana, era affidato l'incarico di «supervisionare, consigliare e sorvegliare» l'attività del governo italiano, specie riguardo l'imposizione dei termini d'armistizio, che richiedevano, fra l'altro, un'attiva partecipazione italiana allo sforzo bellico alleato. L'ACC era divisa in quattro sezioni fortemente irreggimentate: Military, Political, Economic and Administrative e Communications, alle quali rispondevano tante Commissions e Sub-Commissions in proporzione al numero dei ministeri italiani da controllare ${ }^{71}$. Nel suo ruolo di vigile "falco" e protettore degli interessi alleati nei territori restituiti all'autorità italiana, l'ACC esercitava le sue funzioni in virtù dei principi dettati dal controllo indiretto, operando in concerto con il polo del controllo diretto rappresentato dall'AMG.

L'insediamento dell'AMG a Napoli seguì il naturale andamento delle operazioni militari: sin dai giorni immediatamente successivi allo sbarco di Salerno, agli ufficiali dei Civil Affairs erano state impartite istruzioni inerenti lo studio delle future iniziative da intraprendere nella città partenopea. La mattina del 1 ottobre 1943, insieme agli eserciti alleati, entrarono in scena anche i quadri dirigenti di spicco dell'AMGOT: il colonnello Hume, allora al comando della Region III, il tenente colonnello Kraege, neo-insignito CAO di Napoli, e il maggiore Knight guidarono in prima persona le operazioni di requisizione del Municipio, che sancirono, oltre il chiaro aspetto simbolico, lo stanziamento con effetto immediato delle nuove forze occupanti ${ }^{72}$.

Dopo lo sbarco in Sicilia, gli Alleati si apprestavano ad amministrare un'altra grande città italiana, afflitta da ogni tipo di problematiche. Alle privazioni materiali, si aggiungevano il caos e la disgregazione di ogni livello - politico, economico e sociale - della realtà cittadina, provocati dalla guerra e dalla successiva caduta del regime fascista. Governare una città come Napoli avrebbe comportato, prima che il necessario ristabilimento dell'ordine, un'azione minuziosa di ricucimento e ristrutturazione di tutti i gangli vitali della società, giunti alla paralisi e al progressivo deperimento. Un modus operandi che richiedeva innanzitutto una visione ad ampio respiro e proiettata necessariamente nel lungo termine, che spesso confliggeva con l'orizzonte strategico degli ambienti militari, proiettato al conseguimento del maggior numero di risultati in un breve arco temporale. Questo può in parte spiegare le preoccupazioni che investirono gli alti

Italia contemporanea, 136, 1979, pp. 39-66, p. 41.

${ }^{71}$ PORZIO, Maria, op. cit., p. 37.

${ }^{72}$ DE MARCO, Paolo, «Il difficile esordio del governo militare», cit., pp. 42-43. 
comandi alleati circa i primi interventi da adottare e le perplessità di alcuni sottoposti, specialmente quelli impiegati nelle operazioni di polizia, nel dover operare in un teatro ormai lontano dal fronte bellico, che non offriva loro alcuno stimolo né gratificazioni future.

A prima vista Napoli, col tipo di lavoro che ci riserva, sembra poco attraente, in confronto al Nord Africa. I giorni delle scorribande sulle montagne della Cabilia per incontrare i caid ribelli e i santoni che controllano le tribù, e delle discussioni segrete nel roseto dei Giardini di Palazzo a Tunisi, sono finiti per sempre. La vita qui, in confronto, promette di essere faticosa, a volte prosaica, e oppressa dalla routine ${ }^{73}$.

Al contempo, dopo l'iniziale euforia cominciava a farsi largo la consapevolezza che il nuovo regime di occupazione avrebbe richiesto ulteriori sacrifici alla popolazione, in nome delle inappellabili esigenze militari. La presenza sul territorio di innumerevoli comparti alleati, con le annesse ramificazioni burocratico-amministrative al loro seguito, richiedeva innanzitutto una collocazione stabile, nonostante le difficoltà date da un contesto urbano profondamente deficitario sul piano delle abitazioni disponibili. Accanto alle industrie, innumerevoli furono le abitazioni private requisite nel capoluogo. Secondo quanto dichiarato successivamente dal colonello Pennycuick in una conferenza stampa, furono proprio «le necessità militari» a spingere verso l'impopolare occupazione delle abitazioni «in una città nella quale gli alloggi, in seguito alla distruzione apportata dalla guerra, già scarseggiavano». In molti casi, egli aggiunse, furono oggetto di requisizione le case abbandonate, «e gli abitanti che nel 1942 o 1943 erano sfollati verso il Nord non potevano certo aspettarsi di potervi ritornare presto ${ }^{74}$. L'atteggiamento duro e poco incline al dialogo con le autorità italiane e i comitati cittadini condizionava in maniera preponderante i discorsi degli alti ranghi alleati su tale pratica, incentrati principalmente sulla legittimazione della stessa come chiave di un lecito strumento al servizio delle superiori finalità militari. Oltre a costituire un primo segno tangibile dell'occupazione, le requisizioni resero con estrema chiarezza ai civili, e nel modo più spiacevole, che l'agognato processo di pace si attestava ancora in una fase embrionale.

Il ricorso smodato a metodi indiscriminati per la requisizione e la diffusione a macchia d'olio di veri e propri atti illeciti e abusivi - come il mancato rilascio delle dovute ricevute, che rendono tuttora estremamente difficile un computo esatto dei vani requisiti dagli angloamericani ${ }^{75}-$ spinsero le autorità alleate ad emanare, nel maggio 1944, una serie di circolari, concernenti la regolamentazione delle occupazioni dei beni immobili da parte di tutte le agenzie periferiche dell'AMG. Le strutture che non potevano essere requisite senza la prioritaria approvazione dei

\footnotetext{
${ }^{73}$ LEWIS, Norman, op. cit., p. 33.

${ }^{74}$ «Le derequisizioni. Precisazioni del col. Pennycuick», in Il Risorgimento, III, 206, 6 settembre 1945, p. 4.

${ }^{75}$ DE MARCO, Paolo, Polvere di piselli, cit., p. 170.
} 
Comandi generali erano riportate nel seguente ordine: ospedali, scuole, lavanderie, panifici, negozi, depositi di materiale, scorte alimentari o equipaggiamento per l'esercito italiano, teatri, ville dell'area metropolitana di Napoli che non fossero state incluse nelle aree assegnate alle agenzie alleate richiedenti, stadi, parchi pubblici ed edifici di importanza storica e educativa. Le chiese, così come altri luoghi di culto, non potevano essere impiegate per fini non religiosi eccetto che per temporanei ricoveri in casi di emergenza, ma in nessun caso si poteva autorizzare il loro uso per esigenze militari. Secondo accordi con le locali autorità ecclesiastiche veniva ribadito il principio secondo il quale esse rimanevano al servizio degli scopi religiosi di dottrina e fede per le quali erano state consacrati. Le requisizioni dei beni immobili dovevano dunque seguire questo ordine di priorità: edifici abbandonati, edifici pubblici, alberghi, sedi di aziende commerciali, scuole e sedi di istituzioni, case private e appartamenti.

Veniva puntualizzato che, in caso di disponibilità di soluzioni alternative e in mancanza di un'autorizzazione per iscritto da parte dei Comandi generali, non era consentita l'occupazione di edifici di rilevanza storica, come gli archivi e le gallerie d'arte. Il permesso di requisizione ai fini di alloggiamento di truppe non era garantito per le scuole, gli ospedali e altri edifici pubblici: come regola generale era infatti preferibile che queste ultime dimorassero nelle tende piuttosto che nelle abitazioni. L'occupazione degli edifici pubblici era considerata temporanea, in attesa di approvazione di occupazione permanente da parte delle autorità dell'AMG e dell'ACC. Lo sgombero dei proprietari o degli inquilini poteva avere corso solo nel caso di urgenti necessità militari e solo quando nessun' altra soluzione per quello scopo specifico poteva essere ottenuta. Lo sfratto compiuto dai comandi militari era autorizzato nel momento in cui le necessità militari richiedevano l'imperativo dell'immediata occupazione, in caso contrario bisognava richiedere l'intervento dei servizi di assistenza dislocati nel territorio dalle locali autorità alleate. Le esigenze militari e non le convenienze personali dovevano muovere le operazioni di sgombero dei civili dalle proprie abitazioni. Si richiedeva discrezione da parte dei comandi responsabili, in quanto si dovevano considerare le difficoltà a cui andavano incontro i civili sgombrati, soprattutto nelle aree massicciamente distrutte. Era fatto obbligo per tutto il personale impiegato fra le fila degli eserciti alleati di esercitare moderazione e tenere conto dei diritti umani ${ }^{76}$.

Le medesime indicazioni erano già state ribadite in una nota del mese di febbraio, che a sua volta rimandava ad una circolare, redatta l'anno precedente, in materia di allontanamento dei civili dai locali requisiti. Anche qui si riportava che il ricorso allo sgombero era legittimo nei casi di urgenti necessità militari e quando nessun altro locale adatto allo scopo poteva essere utilizzato. All'interno dell'aerea metropolitana di Napoli i comandi di zona dovevano presentare preventivamente una richiesta formale di sgombero all'AMG: non erano dunque consentite

\footnotetext{
${ }^{76}$ ACS, ACC, 10263-115/17, Confidential, Acquisitions of real estate, circular 64, 22th May 1944, bob. 979C, scaf. 189.
} 
operazioni di sfratto autonome condotte dai comandi di zona. Fuori l'area metropolitana di Napoli, l'azione di sfratto diretta attuata dai comandi era consentita nei casi in cui le necessità militari richiedessero l'imperativo di immediata occupazione. Le occupazioni degli edifici che esulavano dalle norme prescritte erano proibite, nonostante fosse sempre presente lo spiraglio costituito da una comprovata situazione emergenziale. Allo stesso tempo, veniva enfatizzato che il criterio da seguire per le requisizioni era la necessità e non la convenienza militare. Si segnalava, al contrario, una tendenza, giudicata apparente dagli scriventi, nel ricorrere allo sfratto laddove le strette necessità militari non erano direttamente coinvolte ${ }^{77}$.

Le frequenti requisizioni suscitavano aspre polemiche e insofferenza diffusa fra la popolazione, sentimenti presenti anche nelle relazioni sulla corrispondenza postale redatte dall'Ufficio informazioni dell'Ispettorato censura militare. Le requisizioni erano percepite in primo luogo come arbitrarie e non indispensabili: «Gli inglesi hanno requisito d'autorità le nostre ville... requisiscono tutto quello può far loro comodo, hanno anche requisito uno stabilimento industriale, recando danni al mobilio e ostacolando così anche la ripresa dell'industria» ${ }^{78}$. A Napoli non mancarono infatti le occupazioni di stabili che travalicarono i dichiarati scopi logistici di governo e controllo del territorio. Accanto agli appartamenti privati dislocati nei quartieri più in vista della città, vennero requisiti locali pubblici, aziende, ospedali, alberghi, teatri, musei, scuole e caserme anche per assicurare maggiori comodità ai militari ivi alloggiati. Come ricordava lo stesso Lewis in merito al signorile edificio assegnato alla Field Security Police:

La Sezione è caduta in piedi. Appena arrivato ho scoperto che ci hanno installato nel Palazzo dei principi di Satriano in Piazza Vittoria, alla fine della Riviera di Chiaia, lo straordinario lungomare di Napoli. Il palazzo, a quattro piani, è una versione napoletana del barocco spagnolo. Noi ne occupiamo il piano nobile, in cima a uno scalone di marmo, con i suoi alti soffitti modanati, i candelabri scintillanti, gli enormi specchi a muro, e un opulento mobilio dorato in stile vagamente Impero. [...] Dalle finestre sulla piazza si vedono gruppi di palme, molte statue e la baia di Napoli. L'FSO [Foreign Service Officer] ci ha sistemato davvero bene ${ }^{79}$.

A partire dal novembre 1943, passarono inoltre in mano alleata siti di straordinaria importanza storico-artistica come il Teatro San Carlo, il Museo Nazionale di San Martino, il Museo Archeologico Nazionale - adibito a deposito di materiali e acquartieramento di truppe -, la Biblioteca Nazionale e quella appartenente all'Istituto Orientale, l'Archivio di Stato a Pizzofalcone, l'Università, il Conservatorio, l'Accademia di Belle Arti, la Reggia di Capodimonte, Castel Nuovo e

\footnotetext{
${ }^{77}$ Ibidem, Civil eviction, circular 27, 10th February 1944.

${ }^{78}$ ACS, Ministero dell'Interno, Gabinetto, 1944-1946, b. 15, f. 1119, Censura militare. Relazioni, Relazione mensile aprile 1945, lettera da Napoli.

${ }^{79}$ LEWIS, Norman, op. cit., p. 33.
} 
il Palazzo Reale ${ }^{80}$; nonché l'Orto botanico, la sede del Banco di Napoli di via Roma, il Palazzo delle Assicurazioni di Piazza Carità, gli uffici della Galleria Umberto I, gli hotel Terminus e Cavour e l'ala ancora integra del Palazzo delle Poste $^{81}$. Alle requisizioni a tappeto dei luoghi-simbolo della millenaria storia napoletana non seguirono le necessarie operazioni cautelari per la tutela degli stessi, come testimoniavano le innumerevoli denunce, presentate dalle autorità italiane e dalla stessa MFAA (Sub-Commission for Monuments, Fine Arts and Archives), riguardanti atti di vandalismo, furti e saccheggi ad opera delle truppe alleate ivi alloggiate.

Il tentativo di ricollocare i soldati in aree prive di oggetti di valore sortì scarsi risultati, anche a causa della bassa considerazione che questi ultimi attribuivano al ruolo degli ufficiali dell'MFAA, etichettati peraltro con l'epiteto dispregiativo di Venus Fixers ("aggiusta-Veneri") ${ }^{82}$. I danni arrecati ai locali del Palazzo Reale, ad esempio, furono talmente gravi dal costringere Badoglio ad avanzare, nell'aprile 1944, in prima persona una protesta formale presso gli alti comandi alleati ${ }^{83}$. Nel riportare la propria testimonianza in merito all'increscioso fenomeno, Lewis non lesinava aspri commenti, spingendosi a puntare il dito su un clima di aperta connivenza, venutosi a creare fra truppe arraffatrici e ufficiali alleati, per la spartizione del "bottino di guerra":

\begin{abstract}
Stanno arrivando reclami per i saccheggi compiuti dalle truppe alleate. In questa guerra, gli ufficiali si sono dimostrati molti più abili della truppa in faccende del genere. Gli ufficiali dei Dragoni della Guardia, cui è toccato l'onore di essere la prima unità britannica a fare il suo ingresso a Napoli, sono stati accusati di aver tagliato le tele dalle cornici nel Palazzo della principessa, e di essersi portati via una raccolta di porcellane di Capodimonte. L'OSS [Office of Strategic Services] ha ripulito la sontuosa dimora di Achille Lauro. Si dice che alcuni dei pezzi più voluminosi siano stati imballati dentro casse per essere spediti in Inghilterra con la connivenza della Marina ${ }^{84}$.
\end{abstract}

La situazione era, al contempo, particolarmente sofferta nella provincia, dove la penuria di edifici agibili nel capoluogo aveva intensificato, da parte alleata, le operazioni di requisizione di stabili privati. Ecco cosa accadeva a Capua nelle parole del rapporto stilato dai carabinieri nell'aprile 1944:

\footnotetext{
${ }^{80}$ COLALUCCI, Sergio, La Sub-Commission for Monuments, Fine Arts and Archives Region III. Il Maggiore Paul Gardner a Napoli, in MIDDIONE, Roberto, PORZIO, Annalisa (a cura di), Napoli 1943. I monumenti e la ricostruzione, Napoli, Edizioni Fioranna, 2010, pp. 54-59, p. 55.

${ }^{81}$ PORZIO, Maria, op. cit., p. 48.

${ }^{82}$ COLALUCCI, Sergio, op. cit., p. 55.

${ }^{83}$ DE MARCO, Paolo, Polvere di piselli, cit., p. 171

${ }^{84}$ LEWIS, Norman, op. cit., p. 41.
} 
Come da ordine dato il 20 corrente del Governatore A.M.G. al sindaco di Capua, gli abitanti dei quattro gruppi di abitazioni civili, delimitati da via Roma, via Francesco Pratillo, via Pomerio e via Santa Maria La Ferrara di detto abitato, sono stati sgombrati nella caserma Pepe. Si ignora quale sia l'esatto motivo dello sgombero, perché tenuto con riserva dalle autorità alleate. Lo sgombero sembra che durerà fino al 10 maggio e è stato effettuato con automezzi e personale alleato. Fra le dette persone regna molto malcontento in vista dei gravi disagi da affrontare ${ }^{85}$.

Nelle campagne del casertano, già duramente provate dalle devastazioni dei tedeschi in ritirata, le requisizioni alleate, che coinvolsero nella maggior parte dei casi terreni adibiti a uso agricolo, provocarono anche ingenti danni al territorio. In un'accorata lettera rivolta al prefetto, il sindaco di Arienzo San Felice si fece interprete della disperazione che attanagliava i cuori e le menti della comunità contadina, a cui era stata inibita la raccolta del grano, alimento indispensabile per il proprio sostentamento ${ }^{86}$. Ulteriori segnalazioni, contenenti esplicite richieste al prefetto affinché si esponesse in qualità di mediatore fra le autorità alleate e la popolazione, provenivano anche dall'area del medio e alto Volturno. Il proprietario di un vasto appezzamento sito fra i comuni di Ailano e Vairano informava la Prefettura circa la cattiva condotta di una divisione di truppe canadesi, le quali, approfittando della posizione isolata e dello scarso controllo operato dai loro ufficiali, si sarebbero abbandonate a furti e razzie di ogni genere ai danni dei contadini ${ }^{87}$. Simili criticità erano riscontrate anche a Pomigliano d'Arco. Una lettera anonima, recante firma di "un gruppo di proprietari e agricoltori", segnalava al prefetto l'intenzione degli inglesi di voler requisire «duemila moggia di fertilissimo terreno coltivato, nella zona estendentisi da Casalnuovo al ponte di Pomigliano d'Arco [...] per essere adibito a deposito di autoveicoli delle forze armate britanniche», privando conseguentemente questi ultimi «di ogni fonte di lavoro produttivo» ${ }^{88}$.

A Barra le requisizioni degli appartamenti privati erano state autorizzate ai fini di alloggiamento dei reduci alleati. Come da prassi, i civili colpiti dalle requisizioni si rivolsero al prefetto, arrivando ad elencare nei minimi particolari l'entità del disagio patito:

Con provvedimento del 2 dicembre 1944, lo scrivente ebbe a subire la requisizione del suo appartamento in Barra al secondo piano della Villa Letizia, a via Giambattista Vela. Contemporaneamente subivano la stessa requisizione gli inquilini del primo e terzo piano. Gli sfrattati per il difetto assoluto di abitazioni locali erano costretti ad arrangiarsi chi in un modo, chi in un altro, ammassando la mobilia di casa, e riducendosi a vivere in condizioni

\footnotetext{
${ }^{85}$ ASNa, Prefettura, Gabinetto, secondo versamento, b. 1256/1, Requisizioni e derequisizioni alleate (1943-1946), Sgombero di popolazione civile da alcuni rioni della città di Capua.

${ }^{86}$ Ibidem, lettera del sindaco di Arienzo San Felice al prefetto di Napoli, Napoli 14 maggio 1944.

${ }^{87}$ Ibidem, lettera del colonnello Nunzio al prefetto di Napoli, Napoli 12 luglio 1944.

${ }^{88}$ Ibidem, lettera anonima al prefetto di Napoli, Napoli 6 giugno 1944.
} 
precarie. Il sacrificio finora sopportato pazientemente è stato assai oneroso, a parte le già tante sofferenze per il passato stato di guerra. Lo scrivente fin dal 7 dicembre 1944 faceva presente il grave danno che ne avrebbe risentito per l'impossibilità di poter esplicare col dovuto decoro la sua professione di avvocato, oltre il grave danno alla mobilia unico suo patrimonio, dopo la distruzione della sua casa a San Giuseppe Vesuviano, bruciata dai tedeschi in ritirata. Lo scrivente, ridotto in due camerette a via Bisignano in Barra, non è in grado neanche di ricevere la sua clientela, ed è anche minacciato di sfratto dall'attuale casetta [...] Poiché risulta che i cinque appartamenti requisiti nella detta villa Letizia a via Giambattista Vela sono detenuti da appena sette o otto militari polacchi, ad uso di pochi uffici, che potrebbero sistemarsi in un sol quarto, poiché lo scrivente e altri requisiti sono in condizioni precarie, si rivolge vivissima istanza a V.S. perché voglia far presente al comando militare alleato le difficili nostre condizioni, e premurare quel Comando-Town Mayor di Portici per la derequisizione delle nostre case ${ }^{89}$.

Sollecitata dalla Prefettura, la Questura di Napoli raccolse le seguenti informazioni: l'appartamento dell'avvocato Doria, requisito dal Comando Town Mayor di Portici, era occupato da militari alleati nel numero di trenta unità, fra ufficiali e soldati ${ }^{90}$. Lo stato di cose si protrasse tuttavia anche l'anno successivo, senza che le locali autorità italiane potessero intervenire in favore della popolazione ${ }^{91}$. La risposta alleata, rappresentata nella persona del colonnello e responsabile del Governo militare a Napoli Pennycuick, all'ulteriore richiesta di chiarimenti da parte del prefetto fu perentoria: «La vostra lettera del 5 maggio gab. 6830 è stata inviata al Town Major di Portici, il quale ha comunicato che l'edificio è adibito a mensa ufficiali dalle truppe polacche, e a meno che non si privino altri civili delle loro case, esso non può essere rilasciato. Per il momento non può essere fissata alcuna data circa la disponibilità dell'edificio stesso» ${ }^{92}$. Lo stesso colonnello fornì a mezzo stampa alcune "precisazioni" riguardo le possibili derequisizioni di locali a uso industriale e abitativo. Dichiarava che le autorità cittadine gli avevano sottoposto una lista di locali il cui rilascio era considerato più urgente, citando per ognuno il numero delle persone che vi potevano essere impiegate. Assicurava infine di aver sottoposto tale lista all'attenzione dei comandi alleati superiori, di modo che, qualora fosse stato permesso dal mutamento della situazione militare, avrebbero potuto essere gradualmente derequisiti ${ }^{93}$.

In un ulteriore incontro, indetto da quest'ultimo con il sindaco di Napoli, il Presidente della Corte d'appello Minervini, il Presidente del Commissariato per gli alloggi Carabona, i

\footnotetext{
${ }^{89}$ Ibidem, lettera dell'avvocato Doria al prefetto di Napoli, Barra 30 giugno 1945.

${ }^{90}$ Ibidem, Questura di Napoli, risposta a nota prefettizia n. 12257 dell'8 luglio 1945, n. prot. 1013479, Napoli 5 agosto 1945.

${ }_{91}^{91}$ Ibidem, lettera di un proprietario al prefetto di Napoli, Napoli 30 aprile 1946.

${ }_{92}$ Ibidem, Headquarters Naples Liaison Group Allied Commission Apo. 794 al prefetto di Napoli, ref. NLG/Bldgs/20/505, Napoli 21 maggio 1946.

${ }^{93}$ «Le derequisizioni. Precisazioni del col. Pennycuick», in Il Risorgimento, III, 206, 6 settembre 1945, p. 4.
} 
rappresentanti del CLN locale, l'Unione Sinistrati e i giornalisti, se da una parte fu costretto ad ammettere la presenza di numerose irregolarità verificatesi durante le operazioni di requisizione degli stabili, dall'altra ribadì fermamente la posizione ufficiale degli Alleati sulla questione:

Durante i primi tempi dell'occupazione alleata, le forze armate furono costrette per impellenti necessità militari a requisire molte case occupate da proprietari o inquilini. Ne risultò che molti furono privati del loro alloggio e le forze militari, per venire loro incontro, diedero loro in molti casi altre abitazioni. Questi alloggi dati in cambio non furono reputati di carattere permanente ma solo una misura provvisoria in casi di emergenza. La responsabilità degli alloggi è dell'autorità italiana. Il prefetto ha, per decreto, il potere di sospendere l'esecuzione di una sentenza di sfratto emessa dalla Corte. L'obbiettivo è quello di concedere un tempo ragionevole allo sfrattato per trovare altra abitazione. Molti inquilini che hanno ricevuto alloggi in cambio dalle autorità alleate sono sotto l'impressione che essi non sono soggetti ad essere sfrattati, e in molti casi presentano lettere ricevute da qualche membro delle forze alleate che dichiarano che sono in legale possesso dell'alloggio. Mentre è in potere del Commissario dell'AMG di sospendere sentenze di sfratto, non è nelle abitudini dell'AMG di usare questo potere se non per necessità militari. Da qui scaturisce il principio dell'AMG che dirette richieste di civili per ottenere la sospensione di una sentenza di sfratto non vengano considerate. Un comandante di un'unità militare può, se considera il caso giustificato, chiedere al Commissario dell'AMG di sospendere lo sfratto. Il Commissario dell'AMG non interverrà se non nei casi enunciati qui sopra ${ }^{94}$.

Veniva inoltre ribadito il fermo divieto per i civili di insediarsi negli stabili abbandonati dalle forze alleate, senza che fosse stato emanato a riguardo un ordine di derequisizione. Tutti i trasgressori sarebbero andati incontro a severe condanne ${ }^{95}$. Il lungo protrarsi delle requisizioni, di cui non si profilava una scadenza nemmeno in tempo di pace, divenne anch'esso oggetto di una crescente insofferenza popolare, la cui esperienza diretta avvertiva maggiormente gli svantaggi e i risvolti oppressivi di una pratica concepita e attuata per l'esclusivo perseguimento degli interessi alleati. La prolungata presenza anglo-americana a Napoli e le successive frizioni diplomatiche scaturite nelle fasi di negoziazione di un trattato di pace per l'Italia finirono infine con l'acuire la percezione che i partner alleati volessero liquidare la questione in maniera impari. Tuttavia, i termini stessi della resa incondizionata sanciti dall'armistizio di Cassibile, se da una parte avevano alimentato le ambizioni dei resistenti italiani nel presentare la lotta di liberazione come un atto di ravvedimento e di completa espiazione dal passato fascista, dall'altra lasciavano allo sconfitto ben pochi margini di negoziazione nella ridefinizione dei futuri assetti postbellici. In

\footnotetext{
${ }^{94}$ «Le derequisizioni. Chiarimenti del col. Pennycuick», in Il Risorgimento, III, 215, 15 settembre 1945, p. 4.

${ }^{95}$ Ibidem.
} 
particolare, la linea strategica britannica aveva premuto fin da subito per il mantenimento di una continuità politico-istituzionale responsabile della fragile permanenza del Regno d'Italia nei territori meridionali ${ }^{96}$.

Non doveva dunque stupire il fatto che, specie nell'epicentro partenopeo, all'insediamento del regime di occupazione alleato nel Mezzogiorno fosse seguita una parallela politica di irrobustimento del blocco dominante di area moderata e filomonarchica, uscito pressoché indenne dai processi di epurazione, il cui crescente protagonismo specie sul fronte dell'emergenza abitativa avrebbe segnato la definitiva esautorazione di ogni rivendicazione concernente un reale mutamento dello status quo prebellico. Il comune desiderio di risollevarsi da un conflitto estremamente duro e sofferto, espresso da ampi strati della popolazione, richiedeva in modo preponderante la tutela dallo spettro della povertà e da un destino incerto. Il perpetrarsi dello stato di insostenibile bisogno, al contrario, acuì i sentimenti di sfiducia e disorientamento da parte di questi ultimi, a cui mancava tutto in tempo di guerra così come in pace.

\footnotetext{
${ }^{96}$ PAVONE, Claudio, Alle origini della Repubblica. Scritti su fascismo, antifascismo e continuità dello Stato, Torino,
} Bollati Boringhieri, 1995, pp. 101-103. 


\section{L'AUTORE}

Martina GARGIULO ha conseguito, nel marzo 2017, la laurea magistrale in Scienze Storiche e Orientalistiche presso l'Università di Bologna. I suoi interessi di ricerca spaziano dalla storia sociale alla storia militare, con un accento particolare sulle dinamiche politico-sociali innescate dal secondo conflitto mondiale e sui rapporti intercorsi fra lo scenario internazionale e i contesti locali dei paesi belligeranti.

URL: < http://www.studistorici.com/progett/autori/\#Gargiulo > 